\title{
BMJ Open Cohort profile: early school years follow-up of the Asking Questions about Alcohol in Pregnancy Longitudinal Study in Melbourne, Australia (AQUA at 6)
}

\author{
Evelyne Muggli (D) ,1,2 Jane Halliday (1) ,2,3 Elizabeth J Elliott, ${ }^{4,5}$ \\ Anthony Penington, ${ }^{2,6}$ Deanne Thompson, ${ }^{1,2}$ Alicia Jane Spittle (D) , 1,7 \\ Della Forster, ${ }^{8,9}$ Sharon Lewis, ${ }^{2,3}$ Stephen Hearps, ${ }^{10}$ Peter J Anderson ${ }^{1,11}$
}

To cite: Muggli E, Halliday J, Elliott EJ, et al. Cohort profile: early school years followup of the Asking Questions about Alcohol in Pregnancy Longitudinal Study in Melbourne, Australia (AQUA at 6). BMJ Open 2022;12:e054706. doi:10.1136/ bmjopen-2021-054706

- Prepublication history and additional supplemental material for this paper are available online. To view these files, please visit the journal online (http://dx.doi.org/10.1136/ bmjopen-2021-054706)

Received 20 June 2021 Accepted 15 December 2021

Check for updates

(C) Author(s) (or their employer(s)) 2022. Re-use permitted under CC BY-NC. No commercial re-use. See rights and permissions. Published by BMJ.

For numbered affiliations see end of article.

Correspondence to

Professor Peter J Anderson; peter.j.anderson@monash.edu

\section{ABSTRACT}

Purpose The Asking Questions about Alcohol in Pregnancy (AQUA) study, established in 2011, is a prebirth cohort of 1570 mother and child pairs designed to assess the effects of low to moderate prenatal alcohol exposure and sporadic binge drinking on long-term child development. Women attending general antenatal clinics in public hospitals in Melbourne, Australia, were recruited in their first trimester, followed up three times during pregnancy and at 12 and 24 months postpartum. The current follow-up of the 6-8-year-old children aims to strengthen our understanding of the relationship between these levels of prenatal alcohol exposure and neuropsychological functioning, facial dysmorphology, brain structure and function.

Participants Between June 2018 and April 2021, 802 of the 1342 eligible AQUA study families completed a parent-report questionnaire (60\%). Restrictions associated with COVID-19 pandemic disrupted recruitment, but early school-age neuropsychological assessments were undertaken with 696 children (52\%), and 482 (36\%) craniofacial images were collected. A preplanned, exposure-representative subset of 146 children completed a brain MRI. An existing biobank was extended through collection of 427 (32\%) child buccal swabs.

Findings to date Over half $(59 \%)$ of mothers consumed some alcohol during pregnancy, with one in five reporting at least one binge-drinking episode prior to pregnancy recognition. Children's craniofacial shape was examined at 12 months of age, and low to moderate prenatal alcohol exposure was associated with subtle midface changes. At 2 years of age, formal developmental assessments showed no evidence that cognitive, language or motor outcome was associated with any of exposure level.

Future plans We will investigate the relationship between prenatal alcohol exposure and specific aspects of neurodevelopment at 6-8 years, including craniofacial shape, brain structure and function. The contribution of genetics and epigenetics to individual variation in outcomes will be examined in conjunction with national and international collaborations.

\section{Strengths and limitations of this study}

- The Asking Questions about Alcohol in Pregnancy cohort study was specifically designed to prospectively collect high-quality data on low to moderate prenatal alcohol exposure and relevant confounders to investigate the risk to offspring neurodevelopment.

- The children are being followed up for the third time at 6-8 years, using sensitive measures of neuropsychological function, 3D craniofacial photography and brain MRI

- A biobank of birth samples and maternal and child buccal DNA enable investigation of the contribution of genetic and epigenetic factors to neurodevelopmental outcomes.

- Despite carefully designed questions, reporting bias will need to be considered in the interpretation of findings, especially around alcohol use.

- The generalisability of some findings will be limited to a general antenatal population of Caucasian women, from middle-income backgrounds and with a low-risk pregnancy.

\section{INTRODUCTION}

Alcohol crosses the placenta and is teratogenic. ${ }^{12}$ Health guidelines around the world, including those developed by the Australian National Health and Medical Research Council, recommend that women who are either pregnant or planning a pregnancy abstain from drinking alcohol. ${ }^{3}$ Alcohol can damage the developing fetal brain through oxidative stress, damage to the mitochondria and interference with the function of growth factors and neurotransmitters as well as through epigenetic changes which regulate gene activity. ${ }^{245}$ The consequences can be devastating at a foundational stage of brain development, ultimately disrupting 
neuronal proliferation and migration and glial functioning. ${ }^{2}$

High levels of alcohol exposure to the fetal brain can cause a spectrum of structural brain abnormalities, facial dysmorphology, neurological problems and neurodevelopmental impairments, collectively termed Fetal Alcohol Spectrum Disorder (FASD) ${ }^{4-8}$ These effects have been replicated in animal models and are undisputed. ${ }^{2}$

Many pregnant women consume some alcohol during pregnancy, especially around the time of conception. ${ }^{9-12}$ This is extremely concerning given the potential harms of prenatal alcohol exposure (PAE) to the developing fetus. Unplanned pregnancy is a common explanation for early pregnancy drinking, particularly for binge drinking exposure. ${ }^{13}$ However, even after pregnancy awareness, a substantial proportion of women continue to drink at low to moderate levels, ${ }^{9}$ sometimes with the knowledge that PAE has the potential to lead to lifelong disabilities in a child. ${ }^{14}$ The lack of convincing evidence of harm from lower levels of $\mathrm{PAE}^{1516}$ and conflicting messages from health professionals concerning adverse effects of low to moderate PAE on the fetus are reasons given by some women for their decision not to abstain. ${ }^{17}$

The effects of PAE vary between individuals likely due to genetic, metabolic, nutritional, social and environmental factors as well as the timing, duration and dose of alcohol. ${ }^{18}$ Human research has provided limited evidence that low to moderate PAE is detrimental to the offspring, with a recent systematic review reporting adverse effects on early child development in six studies, no effect in five studies, and a weak positive effect in two. ${ }^{19}$ The authors concluded that conflicting findings following low PAE may in part be due to a lack of sensitivity for detecting some outcome measures, and inadequate accounting for confounding, environmental and social factors. Since this review was published, a secondary analysis of 9719 children from the Adolescent Brain Cognitive Development Study found that even children with low PAE demonstrated poorer psychological and behavioural outcomes at around 9-10 years of age..$^{20}$ The authors claimed their findings were robust because potential confounding factors were considered, and that stringent demographic matching procedures increased the plausibility of the findings, but while the study's sample size is impressive, collection of exposure and confounder information occurred retrospectively in preadolescence, raising questions around recall and accuracy.

The Asking Questions about Alcohol in Pregnancy (AQUA) prospective cohort study was designed to address the limitations in exposure measurement and collection of confounders, allowing for a robust investigation of the effects of common drinking patterns in pregnancy. ${ }^{21}$

The primary objective of this current follow-up of the cohort (AQUA at 6 ) is to assess neurodevelopment (neuropsychological functioning, brain structure and function and craniofacial shape) in a population-based cohort of children aged 6-8 years with respect to their PAE (none, low, moderate, high or binge level alcohol exposure), taking into account related maternal, child and socioenvironmental factors that may explain individual differences in outcome.

\section{Hypotheses}

1. Any PAE has the possibility of being associated with craniofacial changes (eg, mid-face, nose, lips and eyes), structural brain changes (eg, corpus callosum, basal ganglia, cerebellum) and subtle neuropsychological deficits (eg, motor, attention, executive function, memory and behaviour) at 6-8 years of age.

2. These PAE associations will be influenced by the timing and quantity of alcohol exposure, individual child and maternal characteristics (eg, genetics, nutrition, breastfeeding, maternal mental health) and socioenvironmental factors (eg, education, lifestyle, parenting style).

3. Craniofacial differences at 12 months of age will be associated with outcomes at $6-8$ years, specifically (a) craniofacial shape, (b) brain structure and (c) neuropsychological functioning.

\section{COHORT DESCRIPTION}

The AQUA study comprises a cohort of mother/child dyads recruited from the general population in early pregnancy for longitudinal observation. All women with a singleton pregnancy, attending their first antenatal appointment before 19 weeks gestation, between 25 July 2011 and 30 July 2012, at one of seven public hospital recruitment sites in metropolitan Melbourne, Australia, were eligible to participate. Being 16 years or older and being able to read and write English were prerequisites for participation. The methods are described in detail in the original study protocol. ${ }^{21}$ During pregnancy, women completed three questionnaires, (1) at recruitment $(<18$ weeks' gestation), (2) at 25 weeks' gestation and (3) at 35 weeks' gestation. After birth, questionnaires were sent at 12 and 24 months to women who had completed the three pregnancy questionnaires, and for whom complete PAE information was available $(n=1570)$. An exposure representative subsample of 850 children were sequentially invited to have a 3D craniofacial photo taken at 12 months (517 images taken), and/or a neurodevelopmental assessment using the Bayley Scales of Infant and Toddler Development (Bayley-III) at 24 months (554 assessments completed).

The cohort of children was recruited again aged between 6 and 8 years, for further assessments, including longitudinal 3D analysis of craniofacial shape, state-ofthe-art neuroimaging and standardised neuropsychological measures to assess neurodevelopmental status. Outcome measure details are provided in a dedicated section below.

\section{Study design and procedures}

Of the 1570 mother and child dyads from the original cohort, 55 mothers had withdrawn from the study. We excluded 108 who were lifetime alcohol abstainers 
because our target population was children of mothers who normally drink some alcohol. Another 59 mothers were excluded who could not be classified because they abstained in the first trimester, then averaged an intake of less than one standard drink per week for the remainder of their pregnancy. ${ }^{9}$ Therefore, in the AQUA at 6 follow-up study, 1348 mothers and children were invited to participate. Following the invitation to take part, a further six families were excluded from $A Q U A$ at 6 , because of a recent oncology diagnosis in the child $(n=3)$ or because of a later diagnosed condition impacting longterm development (one child with Down syndrome, one child with Dopa Responsive Dystonia and another child with Sanfilippo Syndrome). The final number of families eligible to participate was 1342.

Data were collected between June 2018 and April 2021.

Neuropsychological assessments and 3D craniofacial imaging were performed in specialist facilities at the Murdoch Children's Research Institute and Royal Children's Hospital (RCH) in Melbourne, Australia. For families unable to travel to the campus, the neuropsychological assessments were administered in the home, at school or another suitable facility such as a library meeting room. Externally assessed test results were obtained when the child had been recently assessed. A PAE-representative subset of children was sequentially invited to have a brain MRI scan, with a target number of 50 in each of three exposure groups: (1) no PAE, (2) PAE in trimester one only and (3) PAE throughout gestation. Primary caregivers (ie, the AQUA study mother in most cases) completed questionnaires online. This questionnaire was also offered to families whose child did not attend a neuropsychological assessment, but who still wished to take part.

For study participation, the neuropsychological assessment and/or questionnaire needed to be completed. All other aspects of the study were optional.

\section{Impact of COVID-19}

Following the COVID-19 pandemic, adaptations to the assessment procedures were necessary to comply with relevant institutional and government guidelines for a safe environment for study participants and assessors. Due to two government-mandated, state-wide lockdowns, face-to-face-assessments were suspended from 17 March to 24 June 2020 and again from 9 July to 20 October 2020. Outside these dates, face-to-face assessments were offered where possible, but with physical distancing measures and hygiene procedures in place to minimise risk of viral transmission. Online telehealth-style assessments via a video conferencing platform were also developed and offered from 12 June 2020, so that families were able to take part while remaining in their own home. The latter involved an abbreviated assessment as certain measures could not be administered using telehealth (eg, movement and coordination items) (online supplemental table 1). Families who took part in the telehealth-style assessment were invited to attend the hospital for a 3D craniofacial photo at a later date with the end to lockdown and when site visits became possible again.

\section{Participation rates}

Of the 1342 eligible families, 802 completed the minimum data required for participation $(60 \%)$ and neuropsychological assessment data are available for 696 children (52\%) (table 1). From commencement of the COVID-19 pandemic, 169 of the assessments were conducted in a telehealth format and another 73 in person with physical distancing in place. Following consent, we obtained externally assessed scores from the family's private psychologist for nine children, which in two instances were complemented by a partial assessment of the remaining tests.

Forty-one children who completed an assessment lived in another state of Australia, 23 of whom were visited by one of our assessors and 18 of whom completed a telehealth-style assessment. Another 14 children who lived overseas completed an assessment, three while visiting Melbourne and 10 via telehealth (data not shown).

Craniofacial photographs were obtained from 482 $(36 \%)$ children. Participation rates in this aspect of the study were significantly impacted by the two Covid19-related lockdown periods where site visits were not possible.

Most of the brain MRIs were obtained prior to the COVID-19 pandemic, with an additional four children able to take part in the time following the lockdowns, resulting in 146 scans (out of a proposed 150) being available for analysis.

Buccal swabs were collected from 427 children, either while attending an in-person assessment or via home collection using a mailed swab kit.

Of 540 eligible families did not take part in AQUA at 6: 308 opted out (23\%); 71 for whom we had no current contact details $(5 \%)$ and 161 who opted out passively either by not responding to any of our follow-ups or after initially expressing interest (12\%).

Compared with those who did not take part, mothers participating in AQUA at 6 were less likely to have been abstinent from alcohol or have smoked tobacco in pregnancy and were also less likely to be under 30 years of age at the time of birth. Mothers taking part were more likely to be tertiary educated at the time of initial recruitment and Caucasian (table 2).

Completion rates of previous postbirth study follow-ups in relation to AQUA at 6 are presented in table 3 .

\section{Exposure assessment}

PAE patterns were assessed in the original AQUA study. ${ }^{9}$ Complete data on drinking frequency, amount and type of alcoholic drink(s) on each occasion were collected for 1570 participants via three questionnaires administered in pregnancy.

\section{Timing of exposure}

Maternal alcohol consumption data were reported for five stages of pregnancy: (1) 3 months before pregnancy; 


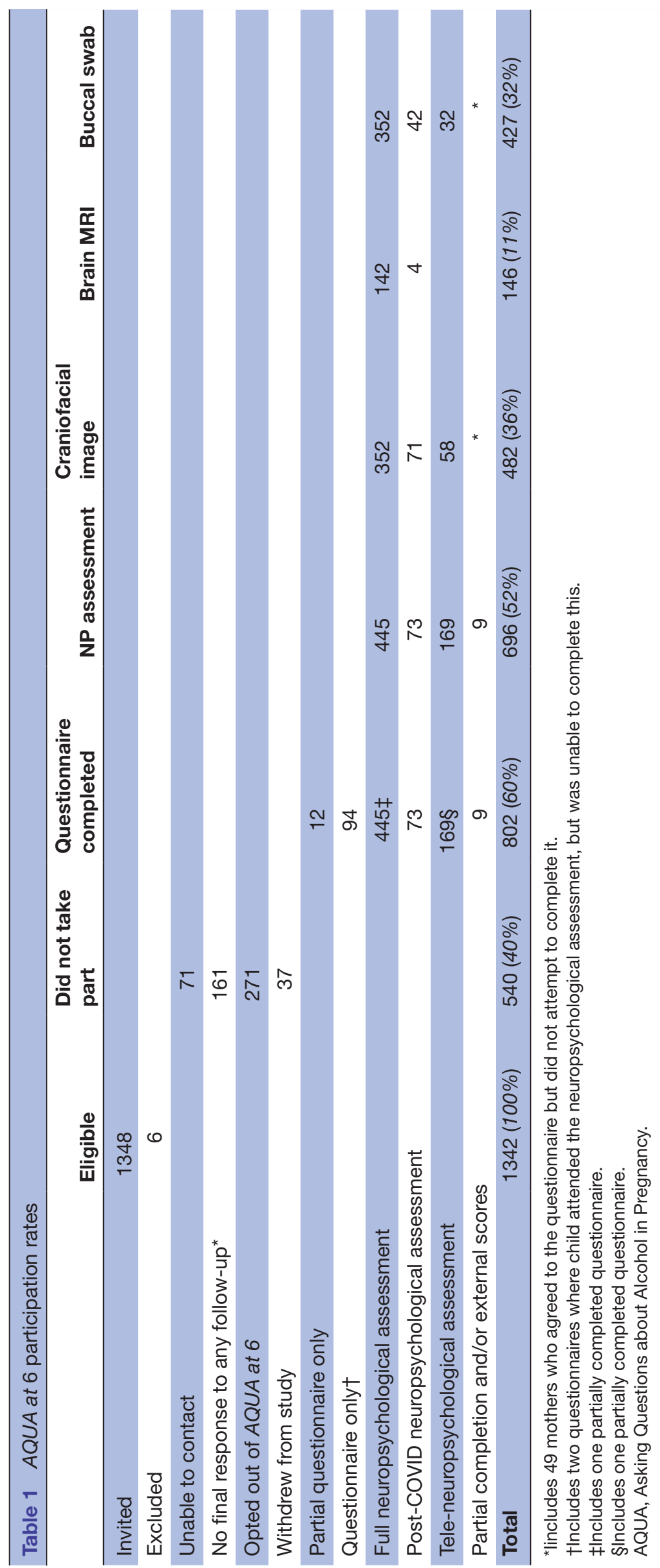

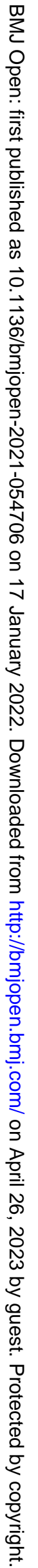


Table 2 Demographics of AQUA at 6 participants compared with those who did not take part

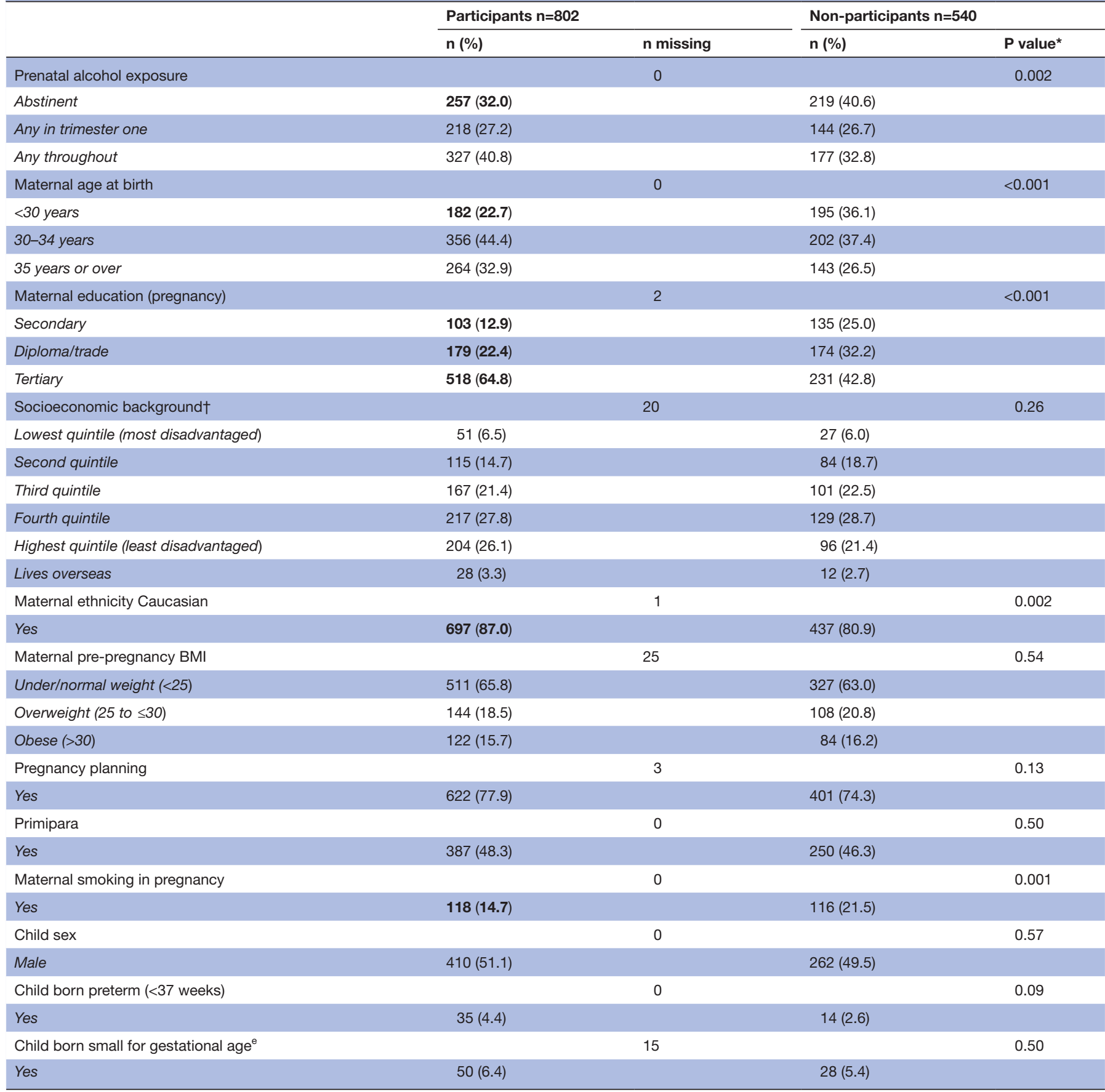

*Pearson's $\chi^{2}$ test; boldface=statistically significant difference between participants and non-participants.

†Based on the Index of Relative Socio-economic Disadvantage: a general socio-economic index summarising and ranking a range of information about the economic and social conditions of people and households within a small geographic area (Statistical Area 1). The index was calculated from the 2016 Census of Population and published by the Australian Bureau of Statistics.

AQUA, Asking Questions about Alcohol in Pregnancy; BMI, body mass index.

(2) trimester 1 prepregnancy aware, (3) trimester 1 postpregnancy aware, (iv) trimester 2 and (v) trimester 3 . The mean (SD) gestational age at pregnancy recognition was 4.9 (1.5) weeks. ${ }^{9}$

Levels of exposure

Women were asked to use a pictorial drinks guide, listing common types and volumes of alcoholic drinks, to identify their 'usual' pattern of drinking, with provision for up to five types of alcoholic drink. For each beverage identified, they were asked how often they usually drank this type of alcohol and how many drinks they usually consumed on each occasion. Women were also asked if there were any 'special occasions' (or difficult times) when they consumed more alcohol than usual, the frequency of these occasions, the drink types and the number of drinks per occasion. Estimates from 'special occasions' 


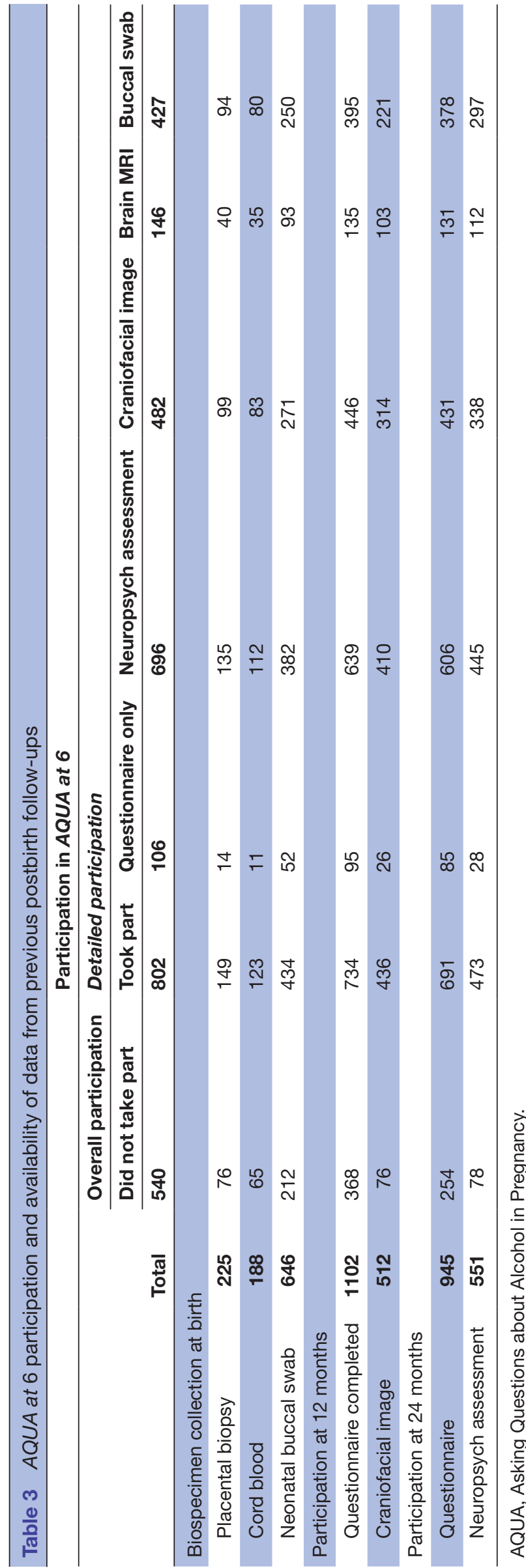

were combined with information from 'usual' alcohol consumption to calculate a maximum weekly intake. ${ }^{9}$ The number and types of drink reported by women were first converted to standard drinks before calculating the amount of absolute alcohol in grams (gAA) consumed. One standard drink in Australia is equal to $10 \mathrm{gAA}$ (online supplemental resource).

Alcohol abstinence throughout pregnancy (but not lifetime abstainer) was defined as the unexposed control group-no PAE.

Summarised exposure group data for the AQUA at 6 eligible cohorts (ie, no PAE; PAE in trimester 1 only; PAE throughout gestation) and participation in the study's core components are presented in table 4. The PAE group distribution in the neuropsychological assessment and 3D craniofacial image data differed marginally from that in the eligible cohort, due to somewhat higher rates of participation in the 'any PAE throughout pregnancy' groups.

In addition to this broad exposure classification, groupbased trajectory modelling (GBTM) will be used as a datadriven method of classifying the temporal, continuous PAE data for all AQUA at 6 analyses. GBTM can be used to objectively identify alcohol consumption trajectories arising directly from the source data without the need for predetermined classification, ${ }^{22}$ which has the potential to result in a more accurate and nuanced representation of the exposure to the fetus.

\section{Outcome measures}

\section{Neuropsychological assessment}

Children underwent a 3-4-hour neuropsychological assessment by trained psychologists blinded to PAE exposure and previous assessments (table 4 ). The measures used were validated, well-established and sensitive to brain insult and were based on measures identified as important to identify neurodevelopmental impairments that are reported in FASD research and included in diagnostic guidelines. ${ }^{723} 24$ They include general intelligence (intelligence quotient; IQ), attention, executive function, memory and learning, language and motor function. Neuropsychological assessments were complemented by information collected via a parent-report questionnaire using validated measures (table 5).

\section{Craniofacial imaging}

Craniofacial imaging of the study child was undertaken by an experienced medical photographer using a 3dMD 7-pod system (3dMD corporation Atlanta, Georgia), which captures a full $360^{\circ}$ image of the head (face and cranium). To ensure that images were unobscured by hair and to capture the shape of the neurocranium, a tightfitting stocking was placed over the cranial vault. Images were captured in less than $1 \mathrm{~s}$ and available for review within $3 \mathrm{~min}$. The photographer and craniofacial image analyst were blinded to the children's PAE.

To represent the entire surface of the cranium and face, a spatially dense array of 69587 points on an age-matched 
Table 4 AQUA at 6 participation by summarised PAE group

\begin{tabular}{|c|c|c|c|c|}
\hline & \multirow[b]{3}{*}{$\mathbf{N}$} & \multicolumn{2}{|c|}{ Exposure group } & \multirow[b]{2}{*}{ Any PAE throughout pregnancy } \\
\hline & & No PAE & Any PAE in trimester 1 only & \\
\hline & & N (\%) & n (\%) & n (\%) \\
\hline Eligible cohort & 1342 & $476(35.5)$ & $362(27.0)$ & $504(37.6)$ \\
\hline Neuropsych assessment & 696 & $223(32.0)$ & $182(26.1)$ & $291(41.8)$ \\
\hline 3D craniofacial image & 482 & $152(31.5)$ & $121(25.1)$ & $209(43.4)$ \\
\hline Brain MRI & 146 & $42(28.8)$ & $45(30.8)$ & $59(40.4)$ \\
\hline Questionnaire only* & 106 & $34(32.1)$ & $36(34.0)$ & $36(34.0)$ \\
\hline
\end{tabular}

*Includes 12 partially completed questionnaires.

AQUA, Asking Questions about Alcohol in Pregnancy; PAE, prenatal alcohol exposure.

template face (pseudo landmarks) is automatically mapped onto each target image by a 3D surface registration algorithm. This warps the shape of the template into the shape of the target face, sampling each face at corresponding locations across the entire surface. A partial least squares regression-based hypothesis testing framework, ${ }^{25}$ suitable for highly multivariate shape data, can be applied to test for group differences, while adjusting for covariates. This can be done on the whole face or on localised facial segments such as the eyes, nose and philtrum. ${ }^{26}$

Brain MRI

Brain imaging was undertaken at the $\mathrm{RCH}$, Melbourne, using a 3 Tesla Siemens MAGNETOM Prisma scanner.
The imaging sequences are listed in table 6 , with total time in the scanner around $45 \mathrm{~min}$. Children in the MRI subgroup were scanned on the same day or within 2 weeks of their neuropsychological assessment. To ensure high compliance and quality images, children completed a preparatory session with a mock MRI scanner prior to their MRI appointment.

Postacquisition MRI analysis includes investigation of: (1) regional brain volumes (66 cortical, 14 subcortical) and cortical morphology (thickness, curvature and sulcal depth) using FreeSurfer V. $7^{27}$; (2) volume and morphology of the corpus callosum, hippocampus, basal ganglia and cerebellum, regions hypothesised to be

\begin{tabular}{|c|c|}
\hline Outcome domain & Scale/subtest \\
\hline \multicolumn{2}{|c|}{ Psychologist assessed (direct assessment) } \\
\hline General intelligence & Core subtests of the Wechsler Intelligence Scale for Children ${ }^{50}$ \\
\hline Language & WISC-V Verbal Comprehension Index ${ }^{50}$ \\
\hline Academic functioning & $\begin{array}{l}\text { Wechsler Individual Achievement Test subtests. Literacy is assessed using the word reading and } \\
\text { spelling subtests, while mathematics is assessed using the numerical operations subtest }{ }^{51}\end{array}$ \\
\hline Attention & $\begin{array}{l}\text { Test of Everyday Attention for Children-Version } 2 \text { subtests (age }<8 \text { years: Balloon Hunt, Barking, } \\
\text { Sustained Attention to Response Task and Simple Reaction Time; age } \geq 8 \text { years: Hector } \\
\text { Cancellation, Vigil, Sustained Attention to Response Task and Simple Reaction Time) }{ }^{52}\end{array}$ \\
\hline Working memory & $\begin{array}{l}\text { Digits Recall, Blocks Recall and Backward Blocks Recall subtests of the Working Memory Test } \\
\text { Battery for Children }\end{array}$ \\
\hline Cognitive flexibility & Contingency Naming Test (trials $1-3)^{5354}$ \\
\hline Episodic memory & The California Verbal Learning Test-Children's version ${ }^{55}$ \\
\hline Motor functioning & Movement Assessment Battery for Children ${ }^{56}$ \\
\hline \multicolumn{2}{|c|}{ Parent report (indirect assessment) } \\
\hline Attention & Attention Deficit Hyperactivity (ADHD) Rating Scale $5^{57}$ \\
\hline $\begin{array}{l}\text { Emotional and } \\
\text { behavioural status }\end{array}$ & Strengths and Difficulties Questionnaire ${ }^{58}$ \\
\hline $\begin{array}{l}\text { Executive function } \\
\text { behaviours }\end{array}$ & Behavior Rating Inventory of Executive Function-Second Edition questionnaire ${ }^{59}$ \\
\hline Autism symptoms & Social Communication Questionnaire ${ }^{60}$ \\
\hline $\begin{array}{l}\text { Movement and } \\
\text { coordination }\end{array}$ & Developmental Coordination Disorder Questionnaire ${ }^{61}$ \\
\hline
\end{tabular}




\section{Table 6 MRI sequences}

1 T1- weighted multi-echo MP-RAGE* images with 0.9 $\mathrm{mm}^{3}$ isotropic voxels (IVs) and echo planar imagenavigated prospective motion compensation

2 Multi-shell simultaneous multi-slice echo planar diffusion images $(b=750,25$ gradient directions; $\mathrm{b}=2000,30$ directions; and $\mathrm{b}=3000 \mathrm{~s} / \mathrm{mm} 2,45$ directions) with $1.5 \mathrm{~mm}^{3} \mathrm{IVs}$ and matching reverse phase encoding sequences

3 3D T2-weighted turbo spin echo images with $0.9 \mathrm{~mm}^{3}$ IVs

4 Multiband, multi-echo gradient recalled echo planar resting state functional MRI images with $2.4 \mathrm{~mm}^{3} \mathrm{IVs}$, with prospective acquisition correction and reverse phase encoding images

*Magnetization Prepared - RApid Gradient Echo

particularly important given previous FASD research ${ }^{1}$; (3) white matter microstructural organisation and maturity using (a) myelin mapping by applying the $T_{1}-T_{2}$ ratio, ${ }^{28}$ (b) the spherical mean technique, which can estimate diffusivity and neurite density from diffusion MRIs without influence from crossing fibres ${ }^{29}$ and (c) fixel-based analyses, a fibre-based analysis of apparent fibre density ${ }^{30} ;(4)$ whole-brain white matter tract analyses using tract-specific analyses $^{31}$ as well as detailed examination of the corpus callosum, anterior-posterior fibre bundles and corticospinal tracts using constrained spherical deconvolution tractography, ${ }^{32}$ given their importance based on previous FASD research ${ }^{33} ;(5)$ structural connectivity is examined using constrained spherical deconvolution-based white matter fibre tractography to find connections between FreeSurfer-derived brain regions. Using graph theory analyses, metrics such as global and local efficiency, small worldness and rich club organisation will be produced to investigate the efficiency, integration or segregation of brain networks ${ }^{34}$ and (6) functional connectivity analyses will also be done by applying independent cocmponent analyses to resting state images, to find temporal correlations in spontaneous blood oxygen level-dependent signal between brain regions. ${ }^{35}$
Australian Early Developmental Census

Consent was sought to link AQUA at 6 children who attended their first year of school in 2018 to the Australian Early Developmental Census (AEDC), ${ }^{36}$ and 93\% of mothers with an eligible child consented to this data linkage. The AEDC is undertaken every 3 years using the Australian version of the Canadian Early Development Instrument, with the most recent year being $2018 .{ }^{37}$ The instrument consists of 100 questions and is completed by teachers on the basis of at least 1 month's knowledge of the child. It covers the five domains of physical, social, emotional, language and cognitive development as well as data on special needs. Children falling below the 10th percentile in any domain are considered developmentally 'vulnerable' in that area, children falling between the 10th and 25th percentile are considered developmentally 'at risk', and all other children are considered to be 'on track'. Approval for linkage to relevant data items at an individual (micro) level will be obtained from the AEDC custodians and the linkage will be conducted independently by an authorised Data Linkage Agency.

\section{Confounders and modifiers}

During the original 2011-2014 AQUA study, extensive data were collected on factors that may confound or modify the relationship between PAE and child outcomes. These included maternal obstetric history and complications, maternal nutrition, medication and supplementation, breastfeeding, maternal and paternal alcohol, tobacco and illicit drug use, maternal mental health, education and other sociodemographics, family relationships and parenting. ${ }^{21}$ Updated relevant information on demographic and socioenvironmental factors was collected from the child's primary caregiver (table 7).

\section{Biospecimens}

A comprehensive biobank of maternal and child DNA from the AQUA study exists, comprising extracts from placental biopsies, cord blood mononuclear cells and maternal and neonatal buccal swabs to investigate how genetic, epigenetic and environmental factors interact with PAE to explain individual variation in child outcomes. ${ }^{21}$ This biobank was extended for future

Table 7 Demographic and socio-environmental factors collected by parent report

\begin{tabular}{ll}
\hline Domain & Questions \\
\hline Demographics & $\begin{array}{l}\text { Ethnic group mother and child, child language spoken at home, mother high school education and post } \\
\text { school training, mother work status, healthcare, financial situation, partner education and work status }\end{array}$ \\
$\begin{array}{l}\text { Child health } \\
\text { Overall health, Child Special Health Care Needs Screener (CSHCN), }{ }^{62} \text { professional assistance and support } \\
\text { Child Rearing Questionnaire, }{ }^{63} \text { Parental Expectations and Limitations, }{ }^{63} \text { Hostile Parenting }{ }^{63}\end{array}$ \\
$\begin{array}{l}\text { Mother health } \\
\text { and lifestyle }\end{array}$ & $\begin{array}{l}\text { Depression Anxiety Stress Scales (DASS-21), }{ }^{64} \text { Alcohol Use Disorder ldentification Test-Consumption } \\
\text { (AUDIT-C), }{ }^{65} \text { tobacco use, illicit substance use }\end{array}$ \\
$\begin{array}{l}\text { Family and } \\
\text { relationships }\end{array}$ & $\begin{array}{l}\text { Marital status, family structure, number of children living in household, McMaster Family Functioning } \\
\text { Subscale, }{ }^{66} \text { family support, }\end{array}{ }^{67}$ couple relationships, ${ }^{67}$ domestic violence, ${ }^{69}$ partner alcohol (AUDIT-C), \\
\end{tabular}


collaborative investigations in this area through the collection of 427 child buccal swabs in 6-8-year olds.

\section{Findings to date}

In the original cohort, there were 1570 mother-child pairs, of whom $59 \%$ of mothers reported drinking alcohol during pregnancy and 19\% reported at least one episode of binge drinking prior to pregnancy recognition. ${ }^{9}$ The study found an association between low to moderate PAE and craniofacial shape in the children aged 12 months, with differences concentrated around the nose, eyes and mouth. ${ }^{38}$ This has potential clinical implications given that development of the face parallels, and is controlled by, the brain. However, at 2 years of age, no adverse association was detected between child neurodevelopment and low to moderate PAE using the Bayley Scales of Infant and Toddler Development (Bayley-III). ${ }^{39}$ Given that measures of early development are only moderately predictive of school-aged outcomes ${ }^{40}$ and do not reliably assess higher order cognitive and motor functions, ${ }^{41}{ }^{42}$ this school-aged follow-up of this cohort is essential to determine any longterm effects of low to moderate PAE and binge episodes.

\section{POWER CALCULATIONS}

Regarding the neuropsychological outcomes, using a twosided 0.05 significance level, with 52\% participation rate, we have $80 \%$ power to detect a small but clinically significant effects (Cohen's $\mathrm{f}=0.12$ ). As an example, for full-scale IQ as effect of this magnitude would translate to a mean difference of 3.6 IQ points between the three major PAE groups. In terms of the MRI data, an important measure of interest is brain volume. Assuming a mean intracranial volume of around 1414 cubic cm (cc) $(\mathrm{SD}=99 \mathrm{cc}),{ }^{43}$ which is based on typically developing 7-year-old children in Melbourne, with a sample size of 146 , we will have $80 \%$ power to detect a difference of $54 \mathrm{cc}$ in total brain volume between groups (medium effect size $\mathrm{f}=0.26$ ). This volumetric reduction represents an effect of $0.54 \mathrm{SD}$ or $4 \%$ of the total volume. Traditional power calculations are not possible for craniofacial analysis, where the outcome measure is many thousands of point coordinates. In our 1-year analysis, we detected differences $(\mathrm{p}<0.05)$ using a control group of 89 and PAE groups of approximately 40 images ${ }^{38}$ which gives us confidence that our proposed analyses will be sufficiently powered.

\section{Strengths and limitations}

This study has several unique features that will enable the relationship between PAE and neurodevelopment to be addressed rigorously. First, AQUA has very detailed assessments of drinking patterns in the periconceptional period and during pregnancy, including timing, frequency and quantity of alcohol consumption. Second, AQUA is a large representative cohort of pregnant women from the community, recruited for the specific purpose of assessing common drinking patterns. Previous studies have tended to focus on high-risk groups of substance users, risking selection bias or take advantage of crude measures collected during large epidemiological studies. ${ }^{16445}$ Third, at each phase of data collection, we have gathered information relating to important modifiers and confounders including nutrition, mental health, physical health and socioeconomic status. Adjustment for these factors is essential given their relationship to both drinking behaviour and child development. Finally, AQUA uses sensitive measures targeting specific areas of neurodevelopment informed by FASD research including longitudinal analysis of craniofacial shape, brain structure and function and neuropsychological functioning. Of importance, our investigations include whether early craniofacial changes are predictive of later neuropsychological impairments, and whether PAE is associated with a common pattern of neural abnormalities demonstrated on MRI.

A limitation of any study measuring PAE is that there are currently no validated objective measures to quantify low to moderate exposure, ${ }^{46}$ and researchers depend on accurate maternal recall and reporting. In order to maximise accuracy of reporting in the AQUA study, we involved pregnant women in the development of the alcohol consumption questions to be used in the AQUA study. ${ }^{47}$ This work indicated that women who attend general antenatal care would answer as truthfully as possible, due to their vested interest in understanding what may be considered normal, non-risky pregnancy drinking habits. The opportunity to report heavy or binge drinking on 'special occasions' yielded important information on early gestation exposures, information that might not have been reported in more general questioning. $^{9}$

The validity of some covariates such as maternal lifestyle and family relationships may also be subjected to reporting bias due to a desire to provide socially acceptable responses. Findings will need to be interpreted in the context of existing literature on the causal relationships between such variables and child neurodevelopment.

Finally, in instances when direct neuropsychological assessment of the child was not possible, we depended on indirect measures (eg, maternal report) to determine developmental progress, which is subjective and may introduce informant bias.

In summary, a significant proportion of pregnant women does not adhere to health policy guidelines and drink some alcohol, potentially putting thousands of children at risk for life-long neurodevelopmental impairments. No safe level of alcohol consumption in pregnancy has been established, and women's drinking behaviour in part reflects the lack of evidence to support health professional advice that women who are pregnant should not drink alcohol. Findings from this study will have an impact from a preventative health perspective, providing strong evidence on the consequences of low to moderate and binge-level PAE, strengthening the messages provided to the public through education and health promotion campaigns. 


\section{COLLABORATIONS}

As with our 12 month follow-up, we are collaborating with two experts in 3D morphometric analysis of image data: Drs Peter Claes and Harold Matthews from KU Leuven in Belgium. The collaboration aims to develop new approaches to undertake our craniofacial analysis and to interpret these results.

Other collaborations to date have arisen from our interest in epigenetics, specifically, the association between PAE and DNA methylation and its role as a mediator of neurodevelopment and FASD. We are contributing data to the Pregnancy And Childhood Epigenetics consortium as part of their meta-analysis project studying early life environmental impacts on human disease using epigenetics. The consortium is based at the US National Institute of Environmental Health Sciences and includes researchers from around the world. (https://www.niehs. nih.gov/research/atniehs/labs/epi/pi/genetics/pace/ index.cfm).

We are also collaborating with the lab of Professor Michael Kobor, Centre for Molecular Medicine and Therapeutics, BC Children's Hospital Research Institute, The University of British Columbia. The Kobor lab recently developed a paediatric epigenetic clock (PedBE) using buccal epithelial swabs (https://github.com/kobor-lab/ Public-Scripts/). The collaboration will generate epigenetic and genotypic data from our child buccal DNA to contribute to their project investigating the extent to which the PedBE clock informs on child development across diverse populations and sex. Another collaboration in this area of study is with a team at the Telethon Institute, Western Australia, led by Dr David Martino. The AQUA study is contributing Epigenome-wide Association study data from buccal epithelial swabs for this project, which aims to identify DNA methylation biomarkers of $\mathrm{PAE}$ in a controlled murine experiment, with replication in existing methylation data sets from human infants with well characterised PAE exposure patters and children diagnosed with FASD (https://www.telethonkids.org.au/ contact-us/our-people/m/david-martino/).

The AQUA study welcomes new collaborations with other investigators and has actively engaged in collaborative data sharing projects. Interested investigators should contact the project manager Evi Muggli (evi.muggli@ mcri.edu.au) to obtain additional information about the study and referral to the appropriate chief investigators for the discussion of collaborative opportunities.

The AQUA study has obtained participant consent to have their data included in other ethically approved studies in related areas of research.

\section{FURTHER DETAILS}

\section{Data management}

All study data are collected and managed using REDCap electronic data capture tools hosted at The Murdoch Children's Research Institute in Melbourne, Australia. ${ }^{48} 49$ REDCap (Research Electronic Data Capture) is a secure, web-based software platform designed to support data capture for research studies, providing (1) an intuitive interface for validated data capture, (2) audit trails for tracking data manipulation and export procedures, (3) automated export procedures for seamless data downloads to common statistical packages and (4) procedures for data integration and interoperability with external sources. REDCap is also used to facilitate tracking and scheduling of all communication with participants. Electronic raw and derived data, including longitudinal data from the first phase of the AQUA study, will be stored on a restricted server and curated by the project manager (EM).

\section{Ethics approval and consent to participate}

All personal information of potential and enrolled participants was collected only for research purpose and will be kept in strict confidentiality by the investigators and project staff. Personal information is stored separately from other research data and will be linked using the family's study ID, which was assigned at enrolment of the pregnant mother in the first phase of the AQUA study. Hard copy materials are kept in locked compartments and electronic records are stored with password encryption. All hard copy and electronic data are stored until child participants are 25 years of age or for 15 years after the study has been completed, whichever is later.

\section{Ethics approval and consent to participate in previous waves of the AQUA study}

Ethical oversight of the cohort's recruitment and prebirth and neonatal follow-ups was provided by the Eastern Health Research and Ethics Committee (E54/1011) and the Human Research Ethics Committees of Mercy Health (R11/14), Monash Health (11071), the Royal Women's Hospital $(11 / 20)$ and the Royal Children's Hospital (31055). The latter also included approval of all procedures pertaining the 12-month and 24-month postpartum follow-ups. Families who have not actively withdrawn their consent to participate are ongoing study participants and their data may be included in future analyses by the project team if they are deemed to be in line with information that was provided to participants at the time of consent.'

\section{Author affiliations}

${ }^{1}$ Victorian Infant Brain Studies, Murdoch Childrens Research Institute, Parkville, Victoria, Australia

${ }^{2}$ Department of Paediatrics, The University of Melbourne, Melbourne, Victoria, Australia

${ }^{3}$ Reproductive Epidemiology, Murdoch Childrens Research Institute, Parkville, Victoria, Australia

${ }^{4}$ Child and Adolescent Health, The University of Sydney, Sydney, New South Wales, Australia

${ }^{5}$ Sydney Children's Hospital Network, Sydney, New South Wales, Australia ${ }^{6}$ Plastic and Maxillofacial Unit, The Royal Children's Hospital, Parkville, Victoria, Australia

${ }^{7}$ Department of Physiotherapy, The University of Melbourne, Melbourne, Victoria, Australia

${ }^{8}$ Judith Lumley Centre, La Trobe University, Melbourne, Victoria, Australia

${ }^{9}$ The Royal Women's Hospital, Parkville, Victoria, Australia

${ }^{10}$ Clinical Sciences, Murdoch Childrens Research Institute, Parkville, Victoria, Australia 
${ }^{11}$ Turner Institute for Brain and Mental Health, Monash University, Clayton, Victoria, Australia

\section{Twitter Alicia Jane Spittle @aliciaspittle}

Acknowledgements The authors would like to thank all AQUA study families who generously gave their time to participate in this study to date. We are extremely grateful to our dedicated staff and students whose enthusiasm never failed despite the COVID-19 pandemic and who recruited and assisted the families through their study participation, undertook the neuropsychological assessments and MRI training and provided feedback to families; Sophie Gibson, Pip Pyman, Garance Delagneau and Drs Stephanie Malarbi, Ngoc Nguyen and Siobhan Seward-Swann. We also thank Stephanie, Ngoc and Siobhan for their invaluable contribution to the COVID-19 related adaptations of the neuropsychological assessment protocols.

Contributors EM, JH, EE, AP, DT, AJS, DF, SL, SH and PJA were involved in the conception and in the design of the study and have made substantial contributions to the planning of the study. EM drafted the manuscript and is responsible for the overall content. EM, JH, EE, AP, DT, AJS, DAF, SL, SH and PJA critically revised manuscript drafts and agreed with the final version of the manuscript.

Funding This work is supported by the National Health and Medical Research Council of Australia (NHMRC) (number 1446635, 2018-2021) and the Victorian State Government's Operational Infrastructure Support Program. DT and AS are supported by NHMRC Career Development Fellowship (Fellowships (APP1160003 to DT, APP1159533 to AS), PA by NHMRC Investigator Grant (APP1176077) and EE by an NHMRC Practitioner Fellowship (GNT1021480) and Medical Research Futures Fund Next Generation Fellowship (MRF1135959).

Competing interests None declared.

Patient consent for publication Not applicable.

Ethics approval This study involves human participants and was approved by Human Research Ethics Committee of the Royal Children's Hospital, Melbourne, Australia, approval number is 38025 . Participants gave informed consent to participate in the study before taking part.

Provenance and peer review Not commissioned; externally peer reviewed.

Data availability statement Data are available upon reasonable request. The datasets generated and/or analysed during the current study will not be publicly available as the study is ongoing. The raw data supporting the conclusions of future manuscripts will be made available to the journal's supplementary material by the authors, if requested. AQUA at 6 study families have the option to consent for their data to be used in future related and ethically approved projects. Following study completion, data will be available from the corresponding author upon reasonable request to such projects.

Supplemental material This content has been supplied by the author(s). It has not been vetted by BMJ Publishing Group Limited (BMJ) and may not have been peer-reviewed. Any opinions or recommendations discussed are solely those of the author(s) and are not endorsed by BMJ. BMJ disclaims all liability and responsibility arising from any reliance placed on the content. Where the content includes any translated material, BMJ does not warrant the accuracy and reliability of the translations (including but not limited to local regulations, clinical guidelines, terminology, drug names and drug dosages), and is not responsible for any error and/or omissions arising from translation and adaptation or otherwise.

Open access This is an open access article distributed in accordance with the Creative Commons Attribution Non Commercial (CC BY-NC 4.0) license, which permits others to distribute, remix, adapt, build upon this work non-commercially, and license their derivative works on different terms, provided the original work is properly cited, appropriate credit is given, any changes made indicated, and the use is non-commercial. See: http://creativecommons.org/licenses/by-nc/4.0/.

\section{ORCID iDs}

Evelyne Muggli http://orcid.org/0000-0002-8102-9811

Jane Halliday http://orcid.org/0000-0001-6206-3857

Alicia Jane Spittle http://orcid.org/0000-0002-6535-661X

\section{REFERENCES}

1 Lebel C, Roussotte F, Sowell ER. Imaging the impact of prenatal alcohol exposure on the structure of the developing human brain. Neuropsychol Rev 2011;21:102-18.
2 Goodlett CR, Horn KH. Mechanisms of alcohol-induced damage to the developing nervous system. Alcohol Res Health 2001;25:175-84.

3 National Health and Medical Research Council. Australian guidelines to reduce health risks from drinking alcohol; guideline 4: pregnancy and breastfeeding. Canberra: NHMRC, 2009.

4 Riley EP, Infante MA, Warren KR. Fetal alcohol spectrum disorders: an overview. Neuropsychol Rev 2011;21:73-80.

5 Bhatia S, Drake DM, Miller L, et al. Oxidative stress and DNA damage in the mechanism of fetal alcohol spectrum disorders. Birth Defects Res 2019;111:714-48.

6 Mattson SN, Crocker N, Nguyen TT. Fetal alcohol spectrum disorders: neuropsychological and behavioral features. Neuropsychol Rev 2011;21:81-101.

7 Bower C. Report to the Australian government department of health: Australian guide to the diagnosis of fetal alcohol spectrum disorder (FASD), 2016.

8 Suttie M, Wozniak JR, Parnell SE, et al. Combined Face-Brain morphology and associated neurocognitive correlates in fetal alcohol spectrum disorders. Alcohol Clin Exp Res 2018;42:1769-82.

9 Muggli E, O'Leary C, Donath S, et al. "Did you ever drink more?" A detailed description of pregnant women's drinking patterns. BMC Public Health 2016;16:683.

10 O'Leary CM, Bower C, Zubrick SR, et al. A new method of prenatal alcohol classification accounting for dose, pattern and timing of exposure: improving our ability to examine fetal effects from low to moderate alcohol. J Epidemiol Community Health 2010;64:956-62.

11 Popova S, Lange S, Probst C, et al. Estimation of national, regional, and global prevalence of alcohol use during pregnancy and fetal alcohol syndrome: a systematic review and meta-analysis. Lancet Glob Health 2017;5:e290-9.

12 Lange S, Probst C, Rehm J, et al. Prevalence of binge drinking during pregnancy by country and world Health organization region: systematic review and meta-analysis. Reprod Toxicol 2017:73:214-21.

13 Rassi A, Wattimena J, Black K. Pregnancy intention in an urban Australian antenatal population. Aust N Z J Public Health 2013;37:568-73.

14 Peadon E, Payne J, Henley N, et al. Attitudes and behaviour predict women's intention to drink alcohol during pregnancy: the challenge for health professionals. BMC Public Health 2011;11:584.

15 Subramoney S, Eastman E, Adnams C, et al. The early developmental outcomes of prenatal alcohol exposure: a review. Front Neurol 2018;9:1108.

16 Mamluk L, Edwards HB, Savović J, et al. Low alcohol consumption and pregnancy and childhood outcomes: time to change guidelines indicating apparently 'safe' levels of alcohol during pregnancy? A systematic review and meta-analyses. BMJ Open 2017;7:e015410.

17 Gibson S, Nagle C, Paul J, et al. Influences on drinking choices among Indigenous and non-Indigenous pregnant women in Australia: a qualitative study. PLoS One 2020;15:e0224719.

18 O'Leary CM, Nassar N, Zubrick SR, et al. Evidence of a complex association between dose, pattern and timing of prenatal alcohol exposure and child behaviour problems. Addiction 2010;105:74-86.

19 Römer P, Mathes B, Reinelt T, et al. Systematic review showed that low and moderate prenatal alcohol and nicotine exposure affected early child development. Acta Paediatr 2020;109:2491-501.

20 Lees B, Mewton L, Jacobus J, et al. Association of prenatal alcohol exposure with psychological, behavioral, and neurodevelopmental outcomes in children from the adolescent brain cognitive development study. Am J Psychiatry 2020;177:1060-72.

21 Muggli E, O'Leary C, Forster D, et al. Study protocol: asking questions about alcohol in pregnancy (aqua): a longitudinal cohort study of fetal effects of low to moderate alcohol exposure. BMC Pregnancy Childbirth 2014;14:302.

22 Nagin DS, Odgers CL. Group-based trajectory modeling in clinical research. Annu Rev Clin Psychol 2010;6:109-38.

23 Wozniak JR, Riley EP, Diagnosis CMD. Epidemiology, assessment, pathophysiology, and management of fetal alcohol spectrum disorders. Lancet Neurol 2019;18:760-70.

24 Hoyme HE, Kalberg WO, Elliott AJ, et al. Updated clinical guidelines for diagnosing fetal alcohol spectrum disorders. Pediatrics 2016;138. doi:10.1542/peds.2015-4256. [Epub ahead of print: 2707 2016].

25 Shrimpton S, Daniels K, de Greef S, et al. A spatially-dense regression study of facial form and tissue depth: towards an interactive tool for craniofacial reconstruction. Forensic Sci Int 2014;234:103-10.

26 Claes P, Roosenboom J, White JD, et al. Genome-wide mapping of global-to-local genetic effects on human facial shape. Nat Genet 2018;50:414-23. 
27 Fischl B, Dale AM. Measuring the thickness of the human cerebral cortex from magnetic resonance images. Proc Natl Acad Sci U S A 2000;97:11050-5.

28 Lee K, Cherel M, Budin F, et al. Early postnatal myelin content estimate of white matter via T1w/T2w ratio. Proc SPIE Int Soc Opt Eng 2015;9417. doi:10.1117/12.2082198. [Epub ahead of print: 1703 2015].

29 Kaden E, Kelm ND, Carson RP, et al. Multi-compartment microscopic diffusion imaging. Neuroimage 2016;139:346-59.

30 Raffelt DA, Tournier J-D, Smith RE, et al. Investigating white matter fibre density and morphology using fixel-based analysis. Neuroimage 2017; 144:58-73.

31 Zhang $\mathrm{H}$, Awate SP, Das SR, et al. A tract-specific framework for white matter morphometry combining macroscopic and microscopic tract features. Med Image Anal 2010;14:666-73.

32 Tournier JD, Calamante F, Connelly A. MRtrix: diffusion tractography in crossing fiber regions. Int J Imaging Syst Technol 2012;22:53-66.

33 Wozniak JR, Muetzel RL. What does diffusion tensor imaging reveal about the brain and cognition in fetal alcohol spectrum disorders? Neuropsychol Rev 2011;21:133-47.

34 Sporns O, Chialvo DR, Kaiser M, et al. Organization, development and function of complex brain networks. Trends Cogn $\mathrm{Sci}$ 2004;8:418-25.

35 Whitfield-Gabrieli S, Nieto-Castanon A. Conn: a functional connectivity toolbox for correlated and anticorrelated brain networks. Brain Connect 2012;2:125-41.

36 Brinkman SA, Gregory TA, Goldfeld S, et al. Data resource profile: the Australian early development index (AEDI). Int J Epidemiol 2014:43:1089-96

37 Janus M, Offord DR. Development and psychometric properties of the Early Development Instrument (EDI): A measure of children's school readiness. Can J Behav Sci 2007;39:1-22.

38 Muggli E, Matthews H, Penington A, et al. Association between prenatal alcohol exposure and craniofacial shape of children at 12 months of age. JAMA Pediatr 2017;171:771-80.

39 Halliday JL, Muggli E, Lewis S, et al. Alcohol consumption in a general antenatal population and child neurodevelopment at 2 years. J Epidemiol Community Health 2017;71:990-8.

40 Roberts G, Anderson PJ, Doyle LW, et al. The stability of the diagnosis of developmental disability between ages 2 and 8 in a geographic cohort of very preterm children born in 1997. Arch Dis Child 2010;95:786-90.

41 Spittle AJ, Spencer-Smith MM, Eeles AL, et al. Does the Bayley-III Motor Scale at 2 years predict motor outcome at 4 years in very preterm children? Dev Med Child Neurol 2013;55:448-52.

42 Spencer-Smith MM, Spittle AJ, Lee KJ, et al. Bayley-II cognitive and language scales in preterm children. Pediatrics 2015;135:e1258-65.

43 Omizzolo C, Thompson DK, Scratch SE. Hippocampal volume and memory and learning outcomes at 7 years in children born very preterm. Journal of the International neuropsychological Society. J Int Neuropsychol Soc 2013;19:1065-75.

44 Mamluk L, Jones T, ljaz S. Evidence of detrimental effects of prenata alcohol exposure on offspring birthweight and neurodevelopment from a systematic review of quasi-experimental studies. Int $J$ Epidemiol 2020;59:1972-95.

45 Roozen S, Peters G-JY, Kok G, et al. Systematic literature review on which maternal alcohol behaviours are related to fetal alcohol spectrum disorders (FASD). BMJ Open 2018;8:e022578.

46 McQuire C, Paranjothy S, Hurt L, et al. Objective measures of prenatal alcohol exposure: a systematic review. Pediatrics 2016;138.

47 Muggli E, Cook B, O'Leary C, et al. Increasing accurate self-report in surveys of pregnancy alcohol use. Midwifery 2015;31:e23-8.
48 Harris PA, Taylor R, Minor BL, et al. The REDCap consortium: building an international community of software platform partners. $J$ Biomed Inform 2019;95:103208

49 Harris PA, Taylor R, Thielke R, et al. Research electronic data capture (REDCap)--a metadata-driven methodology and workflow process for providing translational research informatics support. J Biomed Inform 2009:42:377-81.

50 Wechsler D. Wechsler intelligence scale for children - fifth edition (WISC-V): technical and interpretive manual. Bloomington, MN Pearson, 2014.

51 Breaux KC. Wechsler individual achievement test - third edition (WIAT-III). Technical manual. Bloomington, MN: Pearson, 2009.

52 Manly T, Anderson V, Crawford J. Test of everyday attention for children, second edition (TEA-Ch2). Bloomington, MN: Pearson, 2016.

53 Anderson PJ, Anderson VA, Northam E. Standardization of the contingency naming test (CNT) for school-aged children: a measure of reactive flexibility. Clin Neuropsychol Assess 2000;1:247-73.

54 Taylor HG, Albo VC, Phebus CK, et al. Postirradiation treatment outcomes for children with acute lymphocytic leukemia: clarification of risks. J Pediatr Psychol 1987;12:395-411.

55 Delis DC, Kaplan JH, Kaplan E, et al. California verbal learning test - children's version (CVLT-C). Manual. San Antonio, TX: The Psychological Corporation, 1994

56 Henderson SE, Sugden DA, Barnet AL. Movement assessment battery for children. Second edition (Movement ABC-2): examiner's manual. Bloomington, MN: Pearson, 2007.

57 Lakes KD, Swanson JM, Riggs M. The reliability and validity of the English and Spanish strengths and weaknesses of ADHD and norma behavior rating scales in a preschool sample: continuum measures of hyperactivity and inattention. J Atten Disord 2012;16:510-6.

58 Goodman R. The strengths and difficulties questionnaire: a research note. J Child Psychol Psychiatry 1997;38:581-6.

59 Gioia G, Isquith P, Guy S. BRIEF - behavior rating inventory of executive function. professional manual. Odessa, FL: Psychological Assessment Resources Inc, 2000.

60 Rutter M, Bailey A, Lord C. The social communication questionnaire. Los Angeles, CA: Western Psychological Services, 2003.

61 Wilson BN, Crawford SG, Green D, et al. Psychometric properties of the revised developmental coordination disorder questionnaire. Phys Occup Ther Pediatr 2009;29:182-202.

62 Bethell CD, Read D, Stein REK, et al. Identifying children with specia health care needs: development and evaluation of a short screening instrument. Ambul Pediatr 2002;2:38-48.

63 Wake M, Nicholson JM, Hardy P, et al. Preschooler obesity and parenting styles of mothers and fathers: Australian national population study. Pediatrics 2007;120:e1520-7.

64 Lovibond SH, Lovibond PF. Manual for the depression anxiety stress scales. 2nd edn. Sydney, Australia: Psychology Foundation, 1995.

65 Dawson DA, Grant BF, Stinson FS, et al. Effectiveness of the derived alcohol use disorders identification test (AUDIT-C) in screening for alcohol use disorders and risk drinking in the US general population. Alcohol Clin Exp Res 2005;29:844-54.

66 Epstein NB, Baldwin LM, Bishop DS. The McMaster family assessment DEVICE*. J Marital Fam Ther 1983;9:171-80.

67 Zubrick SR, Smith GJ, Nicholson JM. Social policy research paper No. 34: parenting and families in Australia. Australian Government. Department of Families, Housing, Community Services \& Indigenous Affairs, 2008. ISBN: 978-1-921380-78-5.

68 Ahrons CR. The continuing coparental relationship between divorced spouses. Am J Orthopsychiatry 1981;51:415-28.

69 Gartland D, Hemphill SA, Hegarty K, et al. Intimate partner violence during pregnancy and the first year postpartum in an Australian pregnancy cohort study. Matern Child Health J 2011;15:570-8. 